\title{
Localized modes in capped single-walled carbon nanotubes
}

\author{
Alexander V. Savin ${ }^{1,2, a)}$ and Yuri S. Kivshar ${ }^{1}$ \\ ${ }^{1}$ Nonlinear Physics Center, Research School of Physics and Engineering, Australian National University, \\ Canberra, Australian Capital Territory 0200, Australia \\ ${ }^{2}$ Semenov Institute of Chemical Physics, Russian Academy of Sciences, Moscow 119991, Russia
}

(Received 21 January 2009; accepted 25 February 2009; published online 16 March 2009)

\begin{abstract}
We study numerically small-amplitude oscillations in capped single-walled carbon nanotubes and predict the existence of surface modes localized at capped tips of the nanotubes. These modes are similar to the surface Tamm states found in solids and optics at the edges of truncated lattices. (C) 2009 American Institute of Physics. [DOI: 10.1063/1.3100199]
\end{abstract}

It is well established that a special class of spatially localized modes can be generated at surfaces. ${ }^{1}$ Such surface states have been studied in many branches of physics, including electrons in crystals, ${ }^{2,3}$ surface phonons, ${ }^{4}$ surface polaritons, ${ }^{5}$ and optical surface modes in waveguide arrays. ${ }^{6,7}$ However, a direct observation of highly localized excitations at the atomic level is rather difficult. For example, it took almost 60 years to observe the electronic Tamm states that were originally suggested in $1932 .{ }^{8}$

Carbon nanotubes have attracted considerable attention in recent years, and many of their properties have been observed experimentally. ${ }^{9}$ The growing interest in carbon nanotubes can be explained by their unique physical properties and their potential for a wide range of applications. In particular, carbon nanotubes are known for their superior mechanical strength ${ }^{10}$ and good heat conductance. ${ }^{11}$ Thus, it seems natural to explore a variety of surface-mediated effects in carbon nanotubes, which may acquire additional features in such highly discrete systems with curved geometry and can be subsequently measured experimentally. In particular, the recent experimental studies of the edge structures of thermally treated graphite opened the road for the experimental probing of surface effects. ${ }^{12}$

In this letter we study numerically the surface vibrations of capped single- walled carbon nanotubes and predict the existence of highly localized modes at the tips. These linear localized modes are somewhat similar to the well-known surface Tamm states known in solids.

We consider four types of single-walled carbon nanotubes (SWCNs), which are known to have the smallest diameters. ${ }^{13}$ The structure of the nanotubes is shown in Fig. 1. A zigzag $(m, 0) \mathrm{SWCN}$ with the indices $m=5,6$ has $L$ $\geq 2$ transverse segments consisting of $N=m+2 m L+m$ carbon atoms (each tip has $m$ atoms, and each segment has $2 m$ atoms). The structures with the smallest number of atoms $(N=30$ for $m=5$ and $N=36$ for $m=6)$ at $L=2$ transform into fullerenes $\mathrm{C}_{30}$ (C30.1-D5h) and $\mathrm{C}_{36}$ (No. 15-D6h), respectively.

The armchair nanotube $(m, m)$ with the indices $m=5,6$ has $L \geq 4$ transverse segments with $N=2 m+2 m L+2 m$ carbon atoms (with $2 m$ atoms in the tip and the segment). The structure with the smallest number of atoms $(N=60$ for $m=5$ and $N=72$ for $m=6$ ) for $L=4$ is transformed into fullerenes $\mathrm{C}_{60}$ (bucky-ball C60-Ih) and $\mathrm{C}_{72}$ (C72-D6d), respectively.
To describe oscillations of the nanotube, we present the system Hamiltonian in the form

$$
H=\sum_{n=1}^{N}\left[\frac{1}{2} M\left(\dot{\mathbf{u}}_{n}, \dot{\mathbf{u}}_{n}\right)+P_{n}\right],
$$

where $M=12 \times 1.6603 \times 10^{-27} \mathrm{~kg}$ is the mass of the carbon atom, $\mathbf{u}_{n}=\left[x_{n}(t), y_{n}(t), z_{n}(t)\right]$ is the radius-vector of the $n$th carbon atom at the time $t$. The last term, $P_{n}$, describes the energy of interaction of the $n$th atom with the neighboring atoms,

$$
P_{n}=\frac{1}{2} \sum_{j=1}^{3} V_{j}+\sum_{j=1}^{3} U_{j}+\sum_{j=1}^{3} W_{j} .
$$

The first three terms in Eq. (2) describe the deformation energy due to a direct interaction between pairs of atoms (valent bonds), the next three terms describe the deformation energy of the valent angles, and finally, the last three terms describe the deformation energy associated with a change in the effective angle between the planes created by the valent bonds. The details of the models can be found in Ref. 14 .

In our numerical studies we employ the interaction potentials usually employed for modeling the dynamics of large macromolecules. ${ }^{15-17}$ Namely, for the valent bond coupling we use the potential

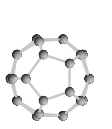

$(5,0)$

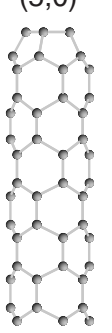

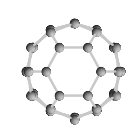

$(6,0)$

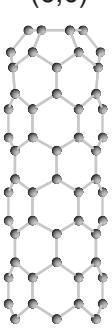

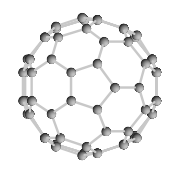

$(5,5)$

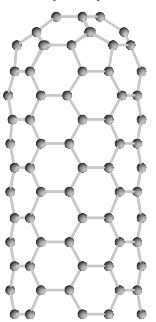

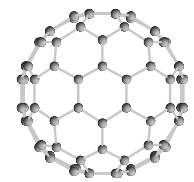

$(6,6)$

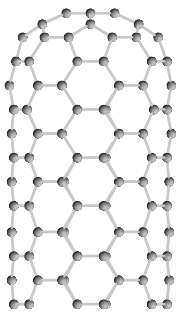

FIG. 1. Structure of the tips of the single-walled capped carbon nanotubes with the chirality indices of $(5,0),(6,0),(5,5)$, and $(6,6)$.

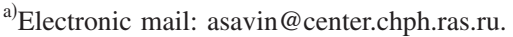




$$
V\left(\mathbf{u}_{1}, \mathbf{u}_{2}\right)=D\left\{\exp \left[-\alpha\left(\rho-\rho_{0}\right)\right]-1\right\}^{2}, \quad \rho=\left|\mathbf{u}_{2}-\mathbf{u}_{1}\right|,
$$

where $D=4.9632 \mathrm{eV}$ is the energy of the valent bond and $\rho_{0}=1.418 \AA$ is an equilibrium length of the bond. For the potential of the valent angle, we take

$$
\begin{aligned}
& U\left(\mathbf{u}_{1}, \mathbf{u}_{2}, \mathbf{u}_{3}\right)=\epsilon_{v}\left(\cos \varphi-\cos \varphi_{0}\right)^{2}, \\
& \cos \varphi=\frac{\left(\mathbf{u}_{3}-\mathbf{u}_{2}, \mathbf{u}_{1}-\mathbf{u}_{2}\right)}{\left|\mathbf{u}_{3}-\mathbf{u}_{2}\right| \cdot\left|\mathbf{u}_{2}-\mathbf{u}_{1}\right|},
\end{aligned}
$$

so that the equilibrium value of the angle is $\cos \varphi_{0}$ $=\cos (2 \pi / 3)=1 / 2$ and the potential of the torsion angle is taken in the form

$$
\begin{aligned}
& W\left(\mathbf{u}_{1}, \mathbf{u}_{2}, \mathbf{u}_{3}, \mathbf{u}_{4}\right)=\epsilon_{t}(1-\cos \phi), \\
& \cos \phi=\left(\mathbf{v}_{1}, \mathbf{v}_{2}\right) /\left(\left|\mathbf{v}_{1}\right| \cdot\left|\mathbf{v}_{2}\right|\right), \\
& \mathbf{v}_{1}=\left(\mathbf{u}_{2}-\mathbf{u}_{1}\right) \times\left(\mathbf{u}_{3}-\mathbf{u}_{2}\right), \\
& \mathbf{v}_{2}=\left(\mathbf{u}_{3}-\mathbf{u}_{2}\right) \times\left(\mathbf{u}_{4}-\mathbf{u}_{3}\right) .
\end{aligned}
$$

The specific values of the parameters $\alpha=1.7889 \AA^{-1}$, $\epsilon_{v}=1.3143 \mathrm{eV}$, and $\epsilon_{t}=0.499 \mathrm{eV}$ are defined from the frequency spectrum of small-amplitude oscillations of a planar graphite. $^{4}$

For comparison, we also study the same model with the interactions described by the Brenner potentials. ${ }^{18,19}$ In this later case, the interaction of the $n$th atom with its three neighbors is described by the sum of three Brenner potentials, $P_{n}=\frac{1}{2}\left(U_{n, 1}+U_{n, 2}+U_{n, 3}\right)$.

To find the ground state of the system, $\left\{\mathbf{u}_{n}^{0}\right\}_{n=1}^{N}$, we solve numerically the minimization problem $E_{0}=\sum_{n=1}^{N} P_{n} \rightarrow \min$. To study small-amplitude oscillations, we apply the expansion $\mathbf{u}_{n}(t)=\mathbf{u}_{n}^{0}+\mathbf{v}_{n}(t)$, where $\left|\mathbf{v}_{n}\right| \ll \rho_{0}$. Then, the equations of motion corresponding to the Hamiltonian Eq. (1) can be presented as a system of $3 N$ linear equations for $3 N$ variables,

$$
-M \ddot{\mathbf{v}}_{n}=\frac{\partial H}{\partial \mathbf{u}_{n}}=\sum_{j=1}^{N} B_{j n} \mathbf{v}_{j}, \quad B_{j n}=\left.\frac{\partial^{2} H}{\partial \mathbf{u}_{n} \partial \mathbf{u}_{j}}\right|_{\left\{\mathbf{u}_{k}^{0}\right\}} .
$$

To find all linear modes of the capped nanotube, we find numerically all $3 N \times 3 N$ eigenvectors and the corresponding eigenvalues of the real symmetric matrix $B=\left(B_{j n}\right)_{j, n=1}^{N}$. If we define $\lambda$ and $\mathbf{e}=\left\{\mathbf{v}_{n}^{0}\right\}_{n=1}^{N}$ as the eigenvalue and normalized eigenvector, namely, $B \mathbf{e}=\lambda \mathbf{e}$ and $(\mathbf{e}, \mathbf{e})=\Sigma_{n}\left(\mathbf{v}_{n}^{0}, \mathbf{v}_{n}^{0}\right)=1$, then the solution of Eq. (6) has the form $\mathbf{v}_{n}(t)=A \mathbf{v}_{n}^{0} \exp (i \omega t)$, where $\omega=\sqrt{\lambda / M}$ is the frequency of the mode and $A$ is its amplitude.

We characterize the spatial localization of an oscillation mode by the parameter of localization (or the inverse participation number) $d=\sum_{n=1}^{N}\left(\mathbf{v}_{n}^{0}, \mathbf{v}_{n}^{0}\right)^{2}$. For the modes that are extended in space, we obtain $d \approx 1 / N$, but for the mode localized at a single atom, we have $d=1$. Dependence of the localization parameter $d$ on the oscillation frequency is shown in Fig. 2. As follows from those figures, each of the four capped nanotubes supports strongly localized surface modes. The number of such localized modes and their degree of localization do not vary with the nanotube length (we consider the lengths $L=25,50,100$, and 200), which indicates that such modes are associated with the capped ends of the nanotubes, being localized at their tips. On the other

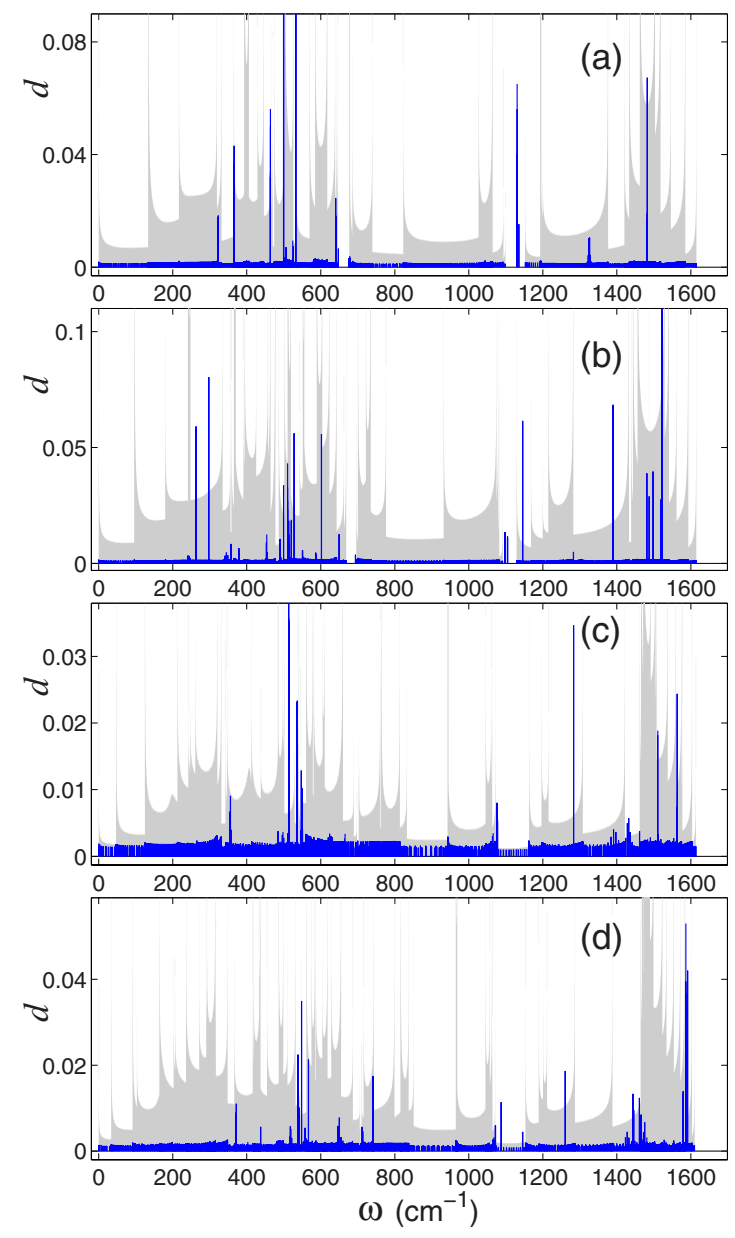

FIG. 2. (Color online) Dependence of the parameter of localization $d$ on the oscillation frequency $\omega$ for the capped nanotubes (a) $(5,0)$, the number of atoms $N=1010$; (b) $(6,0), N=1212$; (c) $(5,5), N=1020$; and (d) $(6,6), N$ $=1224$. Gray shading shows the frequency spectrum density of the corresponding infinite nanotube.

hand, all the other modes can be associated with oscillatory modes of infinite nanotubes (shown in gray in Fig. 2).

More specifically, each tip of the nanotube $(5,0)$ has ten surface modes: four doubly degenerated modes with the frequencies of $\omega=322.9,464.0,499.4$, and $1130.6 \mathrm{~cm}^{-1}$ and two nondegenerated modes with the frequencies of $\omega$ $=538.8$ and $1482.4 \mathrm{~cm}^{-1}$. The nanotube $(6,0)$ has 13 surface modes: one doubly degenerated mode with frequency of $\omega$ $=510.3 \mathrm{~cm}^{-1}$ and 12 modes with frequencies of $\omega=262.9$, 297.5, 499.3, 527.9, 601.9, 1145.7, 1389.6, 1481.0, 1487.2, 1498.1 , and $1522.0 \mathrm{~cm}^{-1}$. The armchair nanotubes demonstrate smaller number of surface modes. Capped nanotube $(5,5)$ has two double modes with frequencies of 513.4 and $536.4 \mathrm{~cm}^{-1}$ and two single modes with frequencies of 532.8 and $1482.4 \mathrm{~cm}^{-1}$. Nanotube $(6,6)$ has one double mode with frequency of $1586.7 \mathrm{~cm}^{-1}$ and five simple modes with frequencies of 538.7, 548.0, 566.7, 1260.5, and $1591.2 \mathrm{~cm}^{-1}$. We have verified that the Brenner potential gives a larger number of localized modes: for the nanotubes $(5,0)$ and $(5,5)$ we obtain 11 modes, and for the nanotubes $(6,0)$ and $(6,6)$ we obtain 22 modes. This difference is explained by the fact that the Brenner potential does not describe adequately highfrequency linear oscillations of the nanotubes, especially in the frequency range of $1600-1700 \mathrm{~cm}^{-1}$, where the surface localized modes are located. 
(a)
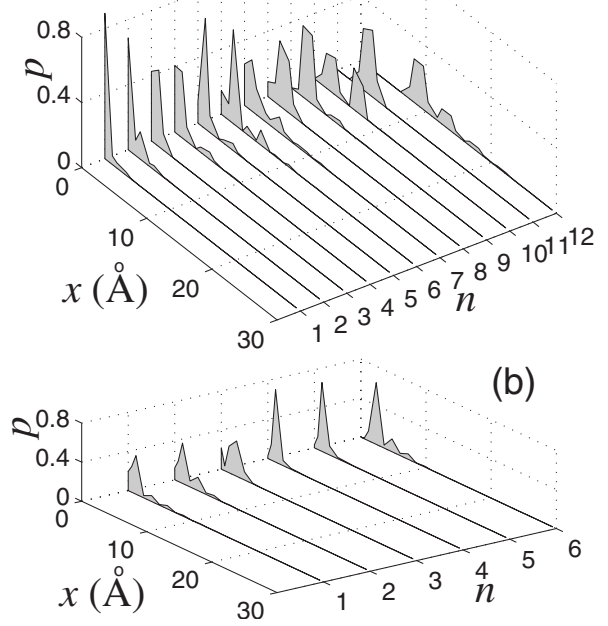

FIG. 3. Distribution of the energy density $p(x)$ of localized modes along the nanotube for the capped nanotubes (a) $(6,0)$ and (b) $(6,6) ; x$ is the distance from the nanotube tip, and $n$ is the mode number. For the nanotube $(6,0)$ the localized modes have frequencies of $\omega=1522.0,527.9,1389.6,601.9,297.5$, $1145.7, \quad 510.3, \quad 1498.1,262.9,499.3, \quad 148.0$, and $1487.2 \mathrm{~cm}^{-1}(n$ $=1,2, \ldots, 12)$. For the nanotube $(6,6)$, the frequencies are $\omega=556.7,538.7$, $1260.5,1591.2,1586.7$, and $548.0 \mathrm{~cm}^{-1}(n=1, \ldots, 6)$.

The largest number of surface localized modes is found in the nanotube $(6,0)$. If we number those modes in accordance with their degree of localization [see Fig. 3(a)], we can divide all the modes into three groups. The first group consists of the modes localized at the very edge of the nanotube (the modes $n=1,2,3,4$ ), which can be related to the oscillations of the hexagon structure of atoms at the tip (see Fig. 1). In-plane oscillations of the hexagon generate the most localized modes $(n=1,2)$. Out-of-plane oscillations involve the neighboring atoms, and the corresponding modes are less localized (modes 3 and 4 ). As a result, the nanotube $(5,0)$ has smaller number of such modes since the tip has only five atoms. The second group of modes (the modes $n=5,6,7,8$ ) is associated with the oscillations of the second transverse segment of atoms, and such oscillations are less localized. Finally, the third group of modes corresponds to the localized oscillations shifted away from the edge of the nanotube (the modes $n=9,10,11,12)$. These modes practically do not involve oscillations of the tip atoms.

Strongly localized modes observed in the nanotube $(6,6)$ are not related directly to the modes of a hexagon at the tip, so the number of such modes is two times smaller. As seen in Fig. 3(b), the oscillations are localized at the two edge segments of atoms and do not penetrate deeper into the structure. Therefore, other types of zigzag nanotubes are expected to support strongly localized surface states.

In addition, we study strongly anharmonic (nonlinear) surface modes localized at the tips. Unlike the linear modes discussed above, which are exact solutions of the linearized equations of motion, the anharmonic modes radiate phonons decaying in a finite time. A nonlinear mode with the largest lifetime that we found is the torsion mode of the nanotube $(6,0)$ with frequency of $527.9 \mathrm{~cm}^{-1}$. For the input kinetic energy of $3.04 \mathrm{eV}$ (mode amplitude $A=70 \AA$ ) and temperature $T=0$, the decay time of this mode (calculated when the mode loses half of its energy) is found to exceed $100 \mathrm{ps,}$ while it is smaller for a finite temperature, e.g., it is 20 ps for $T=30 \mathrm{~K}$ and $9 \mathrm{ps}$ for $T=300 \mathrm{~K}$. Generally, the tips of capped nanotubes may support all types of long-lived anharmonic modes, which generalize the linear modes but demonstrate a finite lifetime of a few picosecond (at room temperatures).

In conclusion, we have studied numerically smallamplitude oscillations of single-walled capped carbon nanotubes and revealed the existence of surface modes localized at the tips of the capped nanotubes in analogy to the surface Tamm states known to exist in solids and optics at the edges of truncated periodic lattices. In the linear regime such modes appear as exact vibrational eigenmodes of the structures, but they also survive in the anharmonic regime and demonstrate long lifetime due to emission of phonons.

A.V.S. acknowledges the hospitality and support of the Nonlinear Physics Centre at the Australian National University where a major part of this work has been completed. This work was supported by the Australian Research Council. The authors also thank the Joint Supercomputer Center of the Russian Academy of Sciences for using computer facilities.

${ }^{1}$ S. G. Davidson and M. Steslicka, Basic Theory of Surface States (Oxford Science, New York, 1996).

${ }^{2}$ I. Tamm, Phys. Z. Sowjetunion 1, 733 (1932).

${ }^{3}$ W. Shockley, Phys. Rev. 56, 317 (1939).

${ }^{4}$ A. A. Maradudin and G. Stegeman, in Surface Phonons, edited by W. Kress and F. W. De Wette (Springer, Berlin, 1991), pp. 5-35.

${ }^{5}$ V. M. Agranovich and D. L. Mills, Surface Polaritons (North-Holland, Amsterdam, 1984).

${ }^{6}$ I. L. Garanovich, A. A. Sukhorukov, and Yu. S. Kivshar, Phys. Rev. Lett. 100, 203904 (2008)

${ }^{7}$ A. Szameit, I. L. Garanovich, M. Heinrich, A. A. Sukhorukov, F. Dreisow, T. Pertsch, S. Nolte, A. Tunnermann, and Yu. S. Kivshar, Phys. Rev. Lett. 101, 203902 (2008).

${ }^{8}$ H. Ohno, E. E. Mendez, J. A. Brum, J. M. Hong, F. Agullo-Rueda, L. L. Chang, and L. Esaki, Phys. Rev. Lett. 64, 2555 (1990).

${ }^{9}$ See, e.g., Physical Properties of Carbon Nanotubes, edited by R. Saito, G. Dresselhaus, and M. S. Dresselhaus (Imperial College Press, London, 1998), and references therein.

${ }^{10}$ M. M. J. Treacy, T. W. Ebbesen, and J. M. Gibson, Nature (London) 381, 678 (1996).

${ }^{11}$ S. Berber, Y.-K. Kwon, and D. Tomanek, Phys. Rev. Lett. 84, 4613 (2000).

${ }^{12}$ Z. Liu, K. Suenaga, P. J. F. Harris, and S. Iijima, Phys. Rev. Lett. 102, 015501 (2009).

${ }^{13}$ H. Y. Peng, N. Wang, Y. F. Zheng, Y. Lifshitz, J. Kulik, R. Q. Zhang, C. S. Lee, and S. T. Lee, Appl. Phys. Lett. 77, 2831 (2000).

${ }^{14}$ A. V. Savin and Yu. S. Kivshar, Europhys. Lett. 82, 66002 (2008).

${ }^{15}$ D. W. Noid, B. G. Sumpter, and B. Wunderlich, Macromolecules 24, 4148 (1991).

${ }^{16}$ B. G. Sumpter, D. W. Noid, G. L. Liang, and B. Wunderlich, Adv. Polym. Sci. 116, 27 (1994).

${ }^{17}$ A. V. Savin and L. I. Manevitch, Phys. Rev. B 58, 11386 (1998); Phys. Rev. E 61, 7065 (2000); Phys. Rev. B 67, 144302 (2003).

${ }^{18}$ D. W. Brenner, Phys. Rev. B 42, 9458 (1990).

${ }^{19}$ D. W. Brenner, O. A. Shenderova, J. A. Harrison, S. J. Stuart, B. Ni, and S. B. Sinnott, J. Phys.: Condens. Matter 14, 783 (2002). 